\title{
The Study on Power Cable Fault Location Based on Wavelet Transform Identification Algorithm
}

\author{
${ }^{1, a}$ Yu De Ming ${ }^{2, b}$ Yang Jing ${ }^{3, c}$ Lu Yi ${ }^{4, d}$ Wang Shu Yuan ${ }^{5, e}$ Li Xin \\ ${ }_{1,2,3,4}$ State Grid North Hebei Electric Power Co., Ltd. ,BeiJing 100000, China \\ ${ }^{5}$ State Grid Zhangjiakou power supply company ,BeiJing 100000, China \\ ayudeming2503@sohu.com bhbdl_yangjing@126.com cluyi_04@sina.com djibei_wangsy@1 \\ 26.com ${ }^{\mathrm{e}}$ xinliss1@163.com
}

Keywords: Wavelet Transform; power cable; fault location; method; study

\begin{abstract}
With the aggravation of power demands, applications of power cables are more extensive. Researchers also start studying power cable fault location to find out a fault point rapidly and accurately, reduce losses effectively, and provide sufficient and continuous power supply. In the paper, by introducing relevant principles of Wavelet Transform, the simulation experiment is conducted. Wavelet decomposition and reconstitution are applied to realize signal filtering. A multi-scale edge detection theory is utilized to realize calculation of cable fault location. The experiment finds that Wavelet Transform is used to calculate power cable fault location with high accurate results. The error is smaller than one sampling distance. Such a method has a larger applied value.
\end{abstract}

\section{Brief Introduction}

With the rapid development of economy, the power demands in various fields are increasingly improved. This promotes the development of power distribution network indirectly and enlarges attention on power cable. In order to ensure various fields have demands for continuous power supply effectively, it is imperative to take measures to reduce power cable faults positively. Under the general trend, power cable fault location is shown in front of the public and becomes a new topic of studies. Traditional power cable fault location mainly applies an offline measurement way, including impedance method, traveling wave method of injecting voltage and once impulse method (details are shown in Table 1). However, in the actual applied and practical process, many defects more or less will be exposed, such as inaccurate power cable fault location, dangerous high-pressure out-point, difficult to reflect traveling wave or weak traveling wave, resulting in failed ranging, and damaged cable insulation's weaknesses, etc. Based on it, it is enough to show that it is hard for power cable fault location to judge power cable faults rapidly and accurately. This impacts timely disposal of faults and can’t ensure reliability of continuous power supply. 
Table 1 Method List of Traditional Power Cable Fault Location

\begin{tabular}{|c|c|c|}
\hline Fault location methods & & Fault properties \\
\hline \multirow{2}{*}{ Classical bridge method } & Low-pressure bridge & Low-resistance fault \\
\hline & Capacitance bridge & Break-line fault \\
\hline Standing wave method & & $\begin{array}{l}\text { Low-resistance and } \\
\text { open-circuit fault }\end{array}$ \\
\hline \multirow[t]{2}{*}{ Impulse method } & $\begin{array}{l}\text { Low-pressure impulse } \\
\text { method }\end{array}$ & $\begin{array}{l}\text { Low-resistance } \\
\text { breakdown, short circuit, } \\
\text { and open-circuit fault }\end{array}$ \\
\hline & $\begin{array}{l}\text { High-pressure impulse } \\
\text { method }\end{array}$ & $\begin{array}{l}\text { High-resistance } \\
\text { breakdown fault }\end{array}$ \\
\hline \multirow{2}{*}{ Flashover method } & Direct flash method & $\begin{array}{c}\text { Flashover high-resistance } \\
\text { fault }\end{array}$ \\
\hline & Impulse flash method & $\begin{array}{l}\text { Leaked high-resistance } \\
\text { and low-resistance fault }\end{array}$ \\
\hline Fault burning method & & High-resistance fault \\
\hline Twice impulse process & & Various faults \\
\hline
\end{tabular}

For this reason, some scholars propose a new method of power cable fault location, namely online location of Wavelet Transformation. Based on Wavelet Transform, it has features of representative signal jumping, and favorable jumping signal processing. It is widely applied to digital signal processing, including smoothing, odd signal detection and signal edge detection. Under different scales, by analyzing signal Wavelet Transform results and extracting signal characteristic parameters, results can be acquired rapidly and accurately. Such a method overcomes shortcomings of traditional location ways in power cable fault location. This will greatly promote the development of power cable fault location, maximize reduction of power cable fault probabilities and provide some safeguard for continuous power supply.

\section{Relevant Overview of Wavelet Transform's Basic Principles}

Main sources of Wavelet Transform are from stretch and translation: Results of stretch can decompose signals under different resolution ratio. The results of translation can regard a unit of signals as windows, so as to observe data information required by detection.

\section{The Basic Definition of Wavelet Transform}

Assuming the function $\Psi(\mathrm{t})$ (L), $\mathrm{L}^{2}(\mathrm{R})$ refers to the square-integrable real number space, namely signal space with limited energy. The Fourier transform is $\hat{\psi}_{\psi}(\omega)$. When $\Psi(\mathrm{w})$ satisfies the conditional expression(1), $\Psi(\mathrm{t})$ is the admissible wavelet, namely basic wavelet or mother wavelet.

$$
C_{\psi}=\int_{-\infty}^{+\infty} \frac{|\hat{\psi}(\omega)|^{2}}{|\omega|} \mathrm{d} \omega<+\infty(1)
$$


A series of sub-functions are generated by stretch and translation through mother wavelet, namely basic wavelet function. In the formula, a is called as a scale factor or stretch factor. The function is to stretch the basic wavelet $\Psi(w)$. The larger a is, the wider ${ }_{\psi}\left(\frac{\mathrm{t}}{\alpha}\right)$ will be. The continuous time of wavelet is broadened with the increase of a, showing a scale (or width) of a function; $b$ is a shift factor. The variable b detects the translation position of a wavelet function on the number axis.

$$
\psi_{\mathrm{a}, \mathrm{b}}(\mathrm{t})=|\mathrm{a}|^{-\frac{1}{2}} \psi\left(\frac{\mathrm{t}-\mathrm{b}}{\mathrm{a}}\right)
$$

$\mathrm{a}, \mathrm{b} \in \mathrm{R}, \mathrm{a} \neq 0$ in Formula(2), it can be observed that the wavelet function has some features, including compact support or approximate compact support and volatility of wavelet in time domains. Besides basic wavelet transform, there are continuous wavelet transform and discrete wavelet transform.

\section{Continuous Wavelet Transform}

It can be observed from the principle of aiming at continuous wavelet transform that the continuous wavelet transform's definition formula for any signal $\mathrm{f}(\mathrm{t})$ is shown in Formula(3):

$$
W_{\mathrm{f}}(\mathrm{a}, \mathrm{b})=\left[\mathrm{f}(\mathrm{t}), \quad \varphi_{\mathrm{a}, \mathrm{b}}(\mathrm{t})\right]=\frac{1}{\sqrt{\mathrm{a}}} \int_{-\infty}^{+\infty} \mathrm{f}(\mathrm{t}) \varphi\left(\frac{\mathrm{t}-\mathrm{b}}{\mathrm{a}}\right) \mathrm{dt}
$$

In the formula (3), by unfolding parameters a and b, frequency spectrum at anytime and any precision can be calculated by the formula.

\section{Discrete Wavelet Transform}

In realistic signal processing process, it is necessary to use a computer to calculate digital signals. Because continuous signals are bad for computer processing, it must use the discrete wavelet. In other words, it must change continuous wavelet into discrete wavelet through discrete. The continuous wavelet transform and discrete wavelet transform are relatively speaking. Discretization just means to discretize a scale factor and shift factor $b$, instead of discretizing time $\mathrm{f}$.

After discretizing the scale factor and shift factor, the following results can be obtained: $a=a_{0}^{n}$ and $b=k_{0}^{n} b_{0}$, so as to obtain the basic function of discrete wavelet:

$$
\varphi_{\mathrm{a}_{0}^{\mathrm{n}}, \mathrm{kb}}=\frac{1}{\sqrt{\mathrm{a}_{0}^{\mathrm{n}}}} \varphi\left(\mathrm{a}_{0}^{-\mathrm{n}} \mathrm{t}-\mathrm{kb}_{0}\right)
$$

Then, a continuous wavelet transform formula is used. Assuming $\mathrm{a}_{0}=2, \mathrm{~b}_{0}=1$, the basic function of binary system wavelet can be further obtained:

$$
\varphi_{2^{\mathrm{n}}, \mathrm{k}}=\frac{1}{\sqrt{2^{\mathrm{n}}}} \varphi\left(2^{-\mathrm{n}} \mathrm{t}-\mathrm{k}\right)
$$

Finally, the discrete wavelet transform of binary system is obtained:

$$
W_{\mathrm{f}}\left(2^{\mathrm{n}}, \mathrm{k}\right)=\frac{1}{\sqrt{2^{\mathrm{n}}}} \int_{-\infty}^{+\infty} \mathrm{f}(\chi) \varphi\left(2^{-\mathrm{n}} \mathrm{t}-\mathrm{k}\right) \mathrm{dt}
$$

\section{Wavelet Transform and Singularity Theory}

Because Wavelet Transform represents local features of signals in the time domain and frequency domain, the position of singularity can be described accurately. Moreover, Moreover, the modulus maximum point and singularity of signals of Wavelet Transform are one-to-one correspondence. Therefore, modulus maximum is used to represent fault information. The modulus maximum of Wavelet Transform is defined as follows: Under a scale a, if for any b, b $\Subset\left(b_{0}+x\right)$, there is $\left|W_{\varphi} \mathrm{f}(\mathrm{a}, \mathrm{b})\right| \leq\left|W_{\varphi} \mathrm{f}\left(\mathrm{a}, \mathrm{b}_{0}\right)\right|$. b0 has local modulus maximum point under the scale a. The local 
modulus maximum is $\left|W_{\varphi} \mathrm{f}\left(\mathrm{a}, \mathrm{b}_{0}\right)\right|$.

\section{The Online Location Simulation Example of Power Cable Faults Based on Wavelet Transform}

Generally speaking, the power cable fault location algorithm of Wavelet Transform has a process, namely start $\rightarrow$ read fault wavelet data $\rightarrow$ decompose and reconstruct 3 scales of the original wave $\rightarrow$ decompose 5-scale wavelet on reconstructive signals $\rightarrow$ detect modulus maximum on individual scales of wavelet decomposition $\rightarrow$ search starting point of positive impulse $\rightarrow$ combine with a computational formula to measure fault distance. Assuming that overall length of a cable is $500 \mathrm{~m}$, fault property is the short circuit. By conducting IICE flashover test EMTP simulation experiment, the cable socket is injected to a long pulse to detect current wave. The wave velocity is $200 \mathrm{~m} / \mathrm{us}$. The sampling frequency is $100 \mathrm{MHz}$ (simulation step is 10ns). The Wavelet Transform is used to obtain 604 and 1104 of discharge pulse's starting point and echo pulse's starting pint. According to wavelet location algorithm, the fault distance calculated is $500 \mathrm{~m}$. It is totally identical to the actual cable length. The specific data figures are shown Figure 1 and Figure 2.

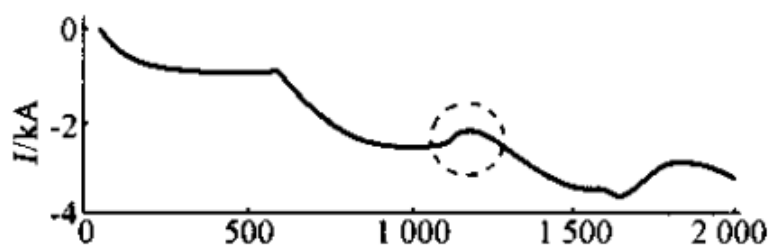

Fig. 1 IICE Simulation Power Cable Fault Location Waveform

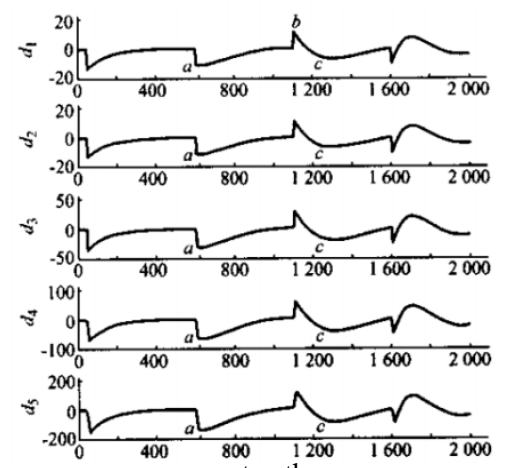

Fig. 2 Modulus Maximum of $1^{\text {st }}-5^{\text {th }}$ Scales of Wavelet Transform

7le fault location.

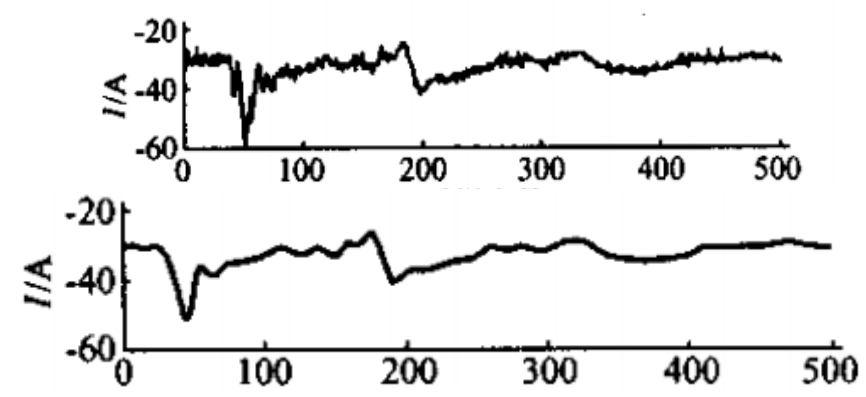

Fig. 3 Power Cable Testing Data and Wavelet Reconstructive Waveform 

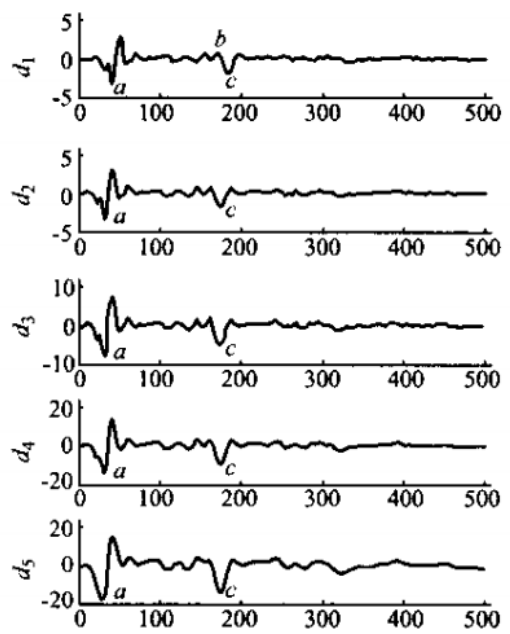

Fig. $41^{\text {st }}-5^{\text {th }}$ Wavelet Coefficients of Reconstructive Data

By detecting the simulation experiment of wavelet location's power cable fault location, it is clear that using wavelet transform can be used to confirm accurate potential fault position. The error is relatively small, so as to provide a large possibility of accuracy for power cable fault location.

\section{References}

[1] Wang Yingli, The Study on Power Cable's Travelling Wave Fault Location Analysis and Fault Positioning[D], Master's Thesis of Xi'an University of Technology, 2012;

[2] Liao Xiaohui, Liang Hengna and Ding Qian, the Study on Power Cable Fault Location Based on Wavelet Transform[J], Journal of Zhengzhou University(Engineering Version), 2013, 34(3): 6-9;

[3] Hu Hamei, The Study on Cable Online Traveling Wave Fault Location Based on Wavelet Transform[D], Master's Thesis of China Three Gorges University, 2009;

[4] Liu Bing and Hu Yong et al., Power Cable Fault Location Based on Wavelet Analysis Frequency Difference[J], Power Automation Equipment, 2009, 29(3): 22-27;

[5] Guo Shanshan and Wang Han, The Simulation Study on Power Cable Fault Location Based on Wavelet Transform[J], Silicon Valley, 2014(20): 40-41;

[6] Jiang Jiaju and Bai Zhiyan, Power Cable Fault Online Location Based on Wavelet Transform[A], High-level Forum Memoir of Chinese Coal Informatization and Automation, 2011;

[7] Li Ming, The Study on Cable Fault Location Based on Wavelet Transform[D], Master's Thesis of Hebei University of Science and Technology, 2009 